\title{
A Case of Esophageal Squamous Cell Cancer in an Endoscopically Normal Esophagus
}

\author{
Ruben Khan ${ }^{\mathrm{a}, \mathrm{c}}$, Mohamed Sultan ${ }^{\mathrm{b}}$, Fuad Maufa ${ }^{\mathrm{b}}$, Nadim Haddad ${ }^{\mathrm{b}}$
}

\begin{abstract}
Esophageal squamous cell carcinoma (ESCC) has traditionally been detected in the later stages of disease which requires aggressive treatment. Newer endoscopic methods have allowed gastroenterologists to detect ESCC lesions and start treatment earlier with the hope to avoid more difficult treatments such as esophagectomy, chemotherapy and radiation. This report follows a patient from detection of ESCC through successful treatment of the ESCC lesions.
\end{abstract}

Keywords: Radiofrequency ablation; Esophagus; Endoscopy; Chromoendoscopy

\section{Introduction}

Esophageal squamous cell carcinoma (ESCC) has been traditionally detected in the later stages of disease requiring aggressive treatment modalities such as esophagectomy, chemotherapy and radiation. Newer endoscopic methods have allowed earlier ESCC detection leading to less invasive and more successful treatment options [1]. The advances allow patients to have more disease-free survival. Furthermore, less invasive treatments avoid some of the complications of the aggressive treatments mentioned previously. This report focuses on the detection, surveillance and treatment of a patient with ESCC in an endoscopically normally appearing esophagus using white light endoscopy (WLE).

\footnotetext{
Manuscript accepted for publication March 4, 2014

a Division of Internal Medicine, MedStar Georgetown University Hospital, 3800 Reservoir Road, NW, Washington DC 20007, USA

${ }^{\mathrm{b}}$ Division of Gastroenterology, MedStar Georgetown University Hospital, 3800 Reservoir Road, NW, Washington DC 20007, USA

${ }^{c}$ Corresponding author: Ruben Khan, Division of Internal Medicine, MedStar Georgetown University Hospital, 3800 Reservoir Road, NW, Washington DC 20007, USA.

Email: Ruben.Khan@gunet.georgetown.edu
}

doi: http://dx.doi.org/10.14740/jmc1724w

\section{Case Report}

A 36-year-old non-smoking female with a history of cervical human papilloma virus was referred to the gastroenterology clinic with an 18-month history of abdominal pain, bloating and intermittent dysphagia. She had similar symptoms 2 years prior when she had an unrevealing esophagogastroduodenoscopy (EGD) at that time. An EGD was performed in our institution which was endoscopically unremarkable using WLE (Fig. 1), and biopsies were obtained from the esophagus to evaluate for eosinophilic esophagitis. Pathology demonstrated high-grade dysplasia (HGD) of the grossly normally appearing esophagus. Given those unexpected results, a repeat EGD was performed which again showed a grossly normal esophagus under WLE. Lugol's solution was then sprayed in the esophagus (known as chromoendoscopy) demonstrating three unstained areas in mid to distal esophagus (Fig. 2). All three areas were biopsied which were positive for HGD/squamous carcinoma in situ. A second pathologist's opinion was obtained which concurred with the findings. Additionally, PCR analysis was positive for human papilloma virus 16 . Chromoendoscopy followed by therapeutic radiofrequency ablation (RFA) was performed. The patient required four sessions over 1 year for complete resolution of the HGD and squamous carcinoma in situ lesions

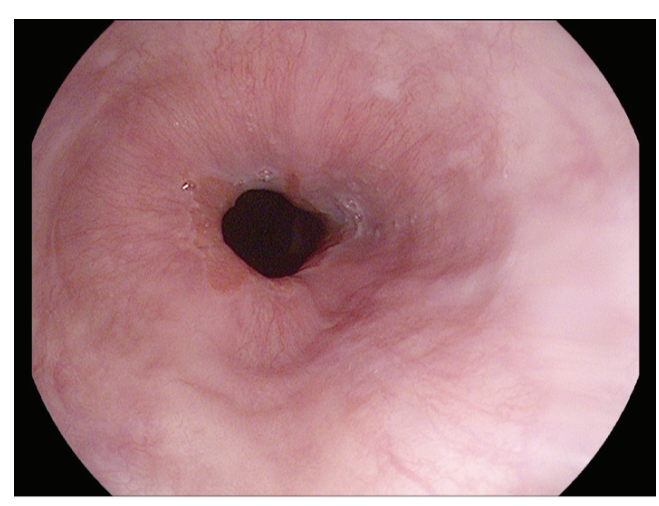

Figure 1. Normal gastroesophageal junction under white light endoscopy. 


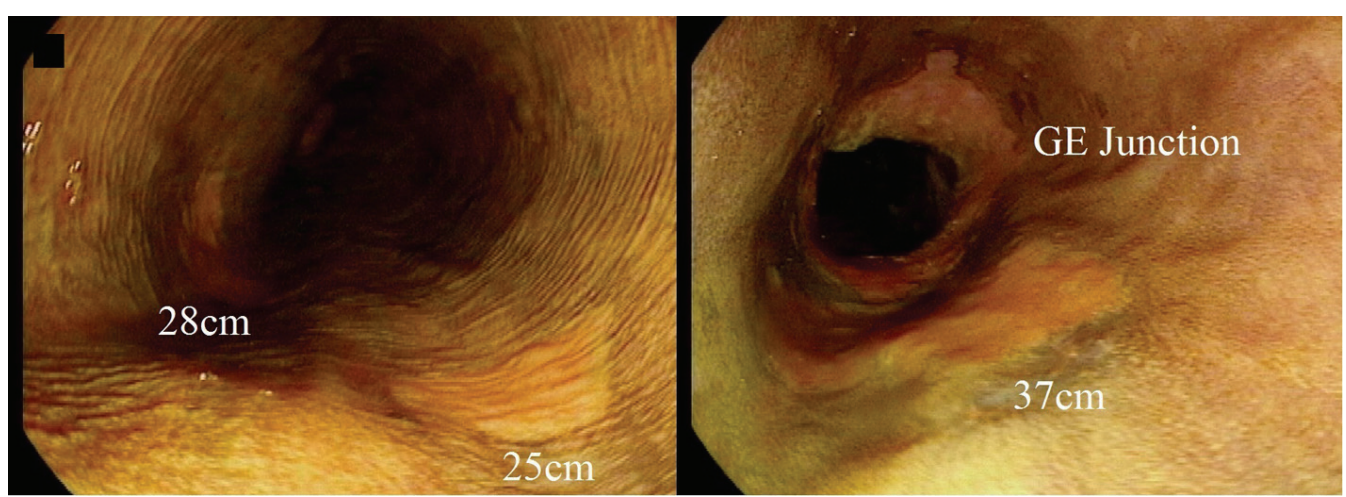

Figure 2. Lugol chromoendoscopy demonstrating ESCC lesions at 25,28 and $37 \mathrm{~cm}$.

(Fig. 3).

\section{Discussion}

This case illustrates the benefit of using chromoendoscopy in detecting dysplasia and ESCC. Since superficial ESCC rarely presents as abnormal tissue under WLE, it is important to have a diagnostic strategy for early ESCC lesions [1]. Lugol chromoendoscopy has been shown to be $92 \%$ sensitive and $94 \%$ specific for detecting intraepithelial squamous cell carcinoma lesions [2]. Unfortunately, Lugol's solution can cause reactions such as chest pain, chest discomfort, esophagitis or iodine shock [1]. Even with these known risks, Lugol chromoendoscopy has been the most widely utilized diagnostic strategy to detect early ESCC lesions.

With the risks of using dyes, there has been a push to create newer forms of non-invasive imaging which could detect early ESCC. Narrow-band imaging (NBI) is a new imaging modality of endoscopy where filters are placed on the endoscope which enhance hemoglobin in small blood vessels [1]. NBI can achieve a high sensitivity and speci-

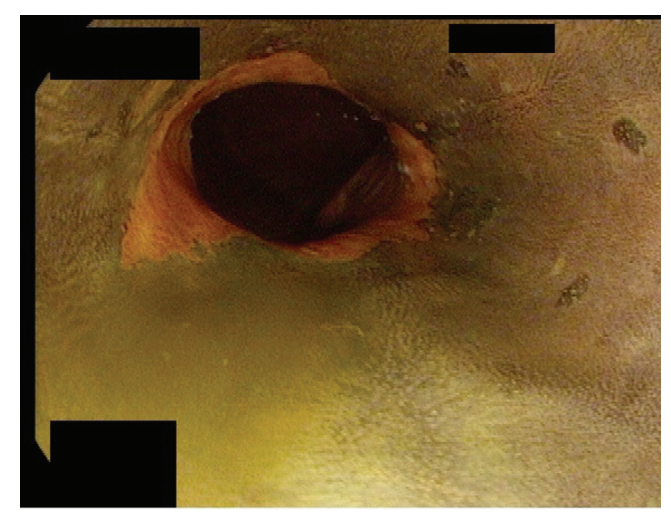

Figure 3. Gastroesophageal junction under Lugol chromoendoscopy after radiofrequency ablation showing resolution of the ESCC lesion. ficity of $97.2 \%$ and $88.9 \%$, respectively $[1,3]$. Autofluorescence imaging (AFI) is yet another non-invasive technique for ESCC screening. AFI produces light excitation of various molecules which can differentiate normal from abnormal tissue. Although it is thought to be fairly sensitive, AFI reaches about $50 \%$ specificity for cancerous lesions. One study improved the specificity of these lesions by normalizing the AFI (NAFI) by obtaining four normalized AFI images. Despite being a small study when compared to traditional AFI, NAFI had a lower sensitivity but higher specificity for malignant lesions. Additionally, this study only included two patients of 12 who had ESCC [4].

There has been a relative paucity of studies which directly compare the various modalities of diagnostic endoscopic methods for ESCC detection. One study compared Lugol chromoendoscopy, NBI, AFI and WLE [5]. Twenty-five lesions were studied and shown under the four modalities to various gastroenterologists. The gold standard of Lugol chromoendoscopy was given a perfect score, and the other modalities were scored against it. NBI had a significantly higher score than WLE or AFI, which had similar scores [5].

Traditionally, ESCC was not detected until advanced stages, requiring more radical therapy such as esophagectomy, chemotherapy and radiation $[6,7]$. For superficial ESCC, endoscopic mucosal resection (EMR) allows a minimally invasive way to remove the ESCC lesion compared to esophagectomy. More recently, endoscopic submucosal dissection (ESD) has been studied as a curative treatment for ESCC. ESD has been compared to EMR, and results from studies thus far demonstrate that ESD has lower rate of local recurrence and higher rate of cure compared with EMR. While both modalities carry a risk of stricture formation, ESD does not cause significantly more perforation than EMR $[8,9]$.

RFA has been recently more widely used for ESCC [10]. RFA is theoretically safer than EMR or ESD since there are no blades used in resection. One study evaluated RFA in patients with superficial esophageal lesions. The lesions were initially identified with Lugol chromoendoscopy, and 
RFA was applied to the lesions. Follow-up Lugol chromoendoscopy determined complete response within 12 months for $97 \%$ of patients [11]. RFA can also be performed more readily as an outpatient procedure while EMR or ESD may require some extent of inpatient monitoring. RFA, however, often needs to be repeated every 2 - 3 months depending on the persistence of the lesions.

In the past, ESCC was diagnosed in more advanced stages requiring esophagectomy, which has higher costs than early detection and surveillance of ESCC [12]. With the use of chromoendoscopy and the newer imaging modalities, it is possible to detect earlier ESCC lesions that frequently looked normal using WLE. Further studies need to elucidate the best modality of screening in the highest-risk populations. RFA is a promising safe and effective treatment modality for ESCC.

\section{Disclosure}

There are neither financial conflicts nor other conflicts of interests with respect to this manuscript. This manuscript did not receive any financial support.

\section{References}

1. Muto M. Endoscopic diagnostic strategy of superficial esophageal squamous cell carcinoma. Dig Endosc. 2013;25(Suppl 1):1-6.

2. Peng G, Long Q, Wu Y, Zhao J, Chen L, Li X. Evaluation of double vital staining with lugol's iodine and methylene blue in diagnosing superficial esophageal lesions. Scand J Gastroenterol. 2011;46(4):406-413.

3. Ishihara R, Takeuchi Y, Chatani R, Kidu T, Inoue T, Hanaoka N, Yamamoto $S$, et al. Prospective evaluation of narrow-band imaging endoscopy for screening of esophageal squamous mucosal high-grade neoplasia in experienced and less experienced endoscopists. Dis Esophagus. 2010;23(6):480-486.
4. Krauss E, Agaimy A, Douplik A, Albrecht H, Neumann H, Hartmann A, Hohenstein R, et al. Normalized autofluorescence imaging diagnostics in upper GI tract: a new method to improve specificity in neoplasia detection. Int J Clin Exp Pathol. 2012;5(9):956-964.

5. Yoshida Y, Goda K, Tajiri H, Urashima M, Yoshimura N, Kato T. Assessment of novel endoscopic techniques for visualizing superficial esophageal squamous cell carcinoma: autofluorescence and narrow-band imaging. Dis Esophagus. 2009;22(5):439-446.

6. Nieman DR, Peters JH. Treatment strategies for esophageal cancer. Gastroenterol Clin North Am. 2013;42(1):187-197.

7. Kranzfelder M, Buchler P, Lange K, Friess H. Treatment options for squamous cell cancer of the esophagus: a systematic review of the literature. J Am Coll Surg. 2010;210(3):351-359.

8. Shimizu Y, Takahashi M, Yoshida T, Ono S, Mabe K, Kato M, Asaka M, et al. Endoscopic resection (endoscopic mucosal resection/ endoscopic submucosal dissection) for superficial esophageal squamous cell carcinoma: current status of various techniques. Dig Endosc. 2013;25(Suppl 1):13-19.

9. Moreira LF, Kamikawa Y, Naomoto Y, Haisa M, Orita $\mathrm{K}$. Endoscopic mucosal resection for superficial carcinoma and high-grade dysplasia of the esophagus. Surg Laparosc Endosc. 1995;5(3):171-175.

10. Zhang YM, Bergman JJ, Weusten B, Dawsey SM, Fleischer DE, Lu N, He S, et al. Radiofrequency ablation for early esophageal squamous cell neoplasia. Endoscopy. 2010;42(4):327-333.

11. Bergman JJ, Zhang YM, He S, Weusten B, Xue L, Fleischer DE, Lu N, et al. Outcomes from a prospective trial of endoscopic radiofrequency ablation of early squamous cell neoplasia of the esophagus. Gastrointest Endosc. 2011;74(6):1181-1190.

12. Gordon LG, Eckermann S, Hirst NG, Watson DI, Mayne GC, Fahey P, Whiteman DC. Healthcare resource use and medical costs for the management of oesophageal cancer. Br J Surg. 2011;98(11):1589-1598. 